\title{
A Real-Time PCR Assay for Detection of Low Pneumocystis jirovecii Levels
}

\begin{abstract}
Susana Ruiz-Ruiz ${ }^{1,2 *}$, Carolina A. Ponce ${ }^{3}$, Nicole Pesantes ${ }^{1,2}$, Rebeca Bustamante ${ }^{3}$, Gianna Gatti ${ }^{3}$, Viviana San Martin ${ }^{4}$, Mireya Gutierrez ${ }^{4}$, Pamela Bórquez ${ }^{4}$, Sergio L. Vargas ${ }^{3}$, Fabien Magne ${ }^{3}$, Enrique J. Calderón ${ }^{2,5}$, Vicente Pérez-Brocal ${ }^{1,2}$ and Andrés Moya ${ }^{1,2,6}$
\end{abstract}

OPEN ACCESS

Edited by:

Olga Matos,

New University of Lisbon, Portugal

Reviewed by: Francisco Esteves,

New University of Lisbon, Portugal Maud Gits-Muselli,

Assistance Publique Hopitaux de Paris, France Francisco Antunes,

Universidade de Lisboa, Portugal

${ }^{*}$ Correspondence: Susana Ruiz-Ruiz ruiz_susrui@gva.es

Specialty section: This article was submitted to Infectious Agents and Disease,

a section of the journal

Frontiers in Microbiology

Received: 30 September 2021 Accepted: 17 December 2021 Published: 11 January 2022

Citation:

Ruiz-Ruiz S, Ponce $C A$ Pesantes N, Bustamante R, Gatti G, San Martin V, Gutierrez M, Bórquez P,

Vargas SL, Magne F, Calderón EJ, Pérez-Brocal $V$ and Moya A (2022) A Real-Time PCR Assay for Detection of Low Pneumocystis jirovecii Levels. Front. Microbiol. 12:787554. doi: 10.3389/fmicb.2021.787554
' Fundación para el Fomento de la Investigación Sanitaria y Biomédica de la Comunitat Valenciana (FISABIO)-Salud Pública, València, Spain, ${ }^{2}$ Centro de Investigación Biomédica en Red en Epidemiología y Salud Pública (CIBEResp), Instituto de Salud Carlos III, Madrid, Spain, ${ }^{3}$ Programa de Microbiología y Micología, Facultad de Medicina, Instituto de Ciencias Biomédicas, Universidad de Chile, Santiago, Chile, ${ }^{4}$ Servicio Médico Legal, Santiago, Chile, ${ }^{5}$ Hospital Universitario Virgen del Rocío, Instituto de Biomedicina de Sevilla, Consejo Superior de Investigaciones Científicas (CSIC), and Universidad de Sevilla, Seville, Spain, ${ }^{6}$ Instituto de Biología Integrativa de Sistemas (I2Sysbio), Universitat de València and Consejo Superior de Investigaciones Cientificas (CSIC), València, Spain

Here we report a new real-time PCR assay using SYBR Green which provides higher sensitivity for the specific detection of low levels of Pneumocystis jirovecii. To do so, two primer sets were designed, targeting the family of genes that code for the most abundant surface protein of Pneumocystis spp., namely the major surface glycoproteins (Msg), and the mitochondrial large subunit rRNA (mtLSUrRNA) multicopy gene, simultaneously detecting two regions. PCR methods are instrumental in detecting these low levels; however, current nested-PCR methods are time-consuming and complex. To validate our new real-time Msg-A/mtLSUrRNA PCR protocol, we compared it with nested-PCR based on the detection of Pneumocystis mitochondrial large subunit rRNA (mtLSUrRNA), one of the main targets used to detect this pathogen. All samples identified as positive by the nested-PCR method were found positive using our new real-time PCR protocol, which also detected $P$. jirovecii in three nasal aspirate samples that were negative for both rounds of nested-PCR. Furthermore, we read both rounds of the nested-PCR results for comparison and found that some samples with no PCR amplification, or with a feeble band in the first round, correlated with higher Ct values in our real-time Msg-A/mtLSUrRNA PCR. This finding demonstrates the ability of this new single-round protocol to detect low Pneumocystis levels. This new assay provides a valuable alternative for $P$. jirovecii detection, as it is both rapid and sensitive.

Keywords: real-time PCR, low P. jirovecii loads, major surface glycoproteins, mitochondrial large subunit rRNA, simultaneous detection

\section{INTRODUCTION}

Pneumocystis jirovecii is an opportunistic fungal pathogen. Children represent one of the main reservoirs in humans but predominantly suffer subclinical infection; therefore, $P$. jirovecii detection is hindered given the absence of overt signs or symptoms (Morris et al., 2008). In this context, PCR methods are instrumental in detecting low $P$. jirovecii levels, as colonization is characterized by a 
low fungal burden in the respiratory tract, hampering its detection. Several PCR and real-time PCR assays have been standardized targeting different single-copy genes such as superoxide dismutase ( $\operatorname{sod}$ ) (Morilla et al., 2019), dihydrofolate reductase (dhfr) (Alanio et al., 2016), cytochrome c oxidase (cox1 ), or the kexin-like serine protease (kex1) gene (Dunaiski et al., 2018). Increased detection sensitivity has also been reported using multicopy genes, such as the mitochondrial large subunit ribosomal RNA ( $m t L S U r R N A$ ) (Morris et al., 2008; Alanio et al., 2011; Fraczek et al., 2019; Chotiprasitsakul et al., 2020; Yang et al., 2020), the mitochondrial small subunit ribosomal RNA ( $m$ tSSUrRNA) (Dellière et al., 2019) or the major surface glycoprotein (Msg) gene family (Rudramurthy et al., 2018; Bossart et al., 2020; Gits-Muselli et al., 2020). In addition, multiplex real-time PCR methods have also been developed where more than one gene is detected to characterize $P$. jirovecii specific single-nucleotide polymorphisms located at different loci (Esteves et al., 2011; Montesinos et al., 2017). However, to date, the nested-PCR targeting the Pneumocystis mitochondrial large subunit rRNA ( $m$ tLSUrRNA) remains one of the most widely used techniques for detecting $P$. jirovecii at low levels. NestedPCR consists of two sequential rounds of conventional PCR, in which the second round of PCR amplifies an internal region of the amplicons generated in the first round (Wakefield et al., 1990; Wakefield, 1996). The two rounds of PCR make this technique highly sensitive, enabling the detection of low fungal burdens (Ponce et al., 2010; Özkoç et al., 2016). This nestedPCR is the method of choice for detecting low P. jirovecii loads in non-invasive samples such as oral lavage, nasopharyngeal aspirates, or in lung samples. However, this method is laborious, time-consuming, and entails considerable risk of contamination, increasing the risk of detecting false positives (Chabé et al., 2014). By contrast, real-time PCR has several advantages, including relatively rapid assay times (hours), reliability, and ease of replicating analyses.

Previous studies have attempted to use the burden of $P$. jirovecii to differentiate between acceptable levels of colonization and those where $P$. jirovecii pneumonia $(\mathrm{PcP})$ represents a health risk (Rudramurthy et al., 2018). However, even if standardized underlying causes of immunosuppression are met, the Pneumocystis burden in itself is not specific enough to define PcP. Indeed, there is a gray zone with burden overlapping where $\mathrm{PcP}$ and colonization cases coexist (RobertGangneux et al., 2014; Fauchier et al., 2016; Sarasombath et al., 2021). Therefore, more precise information on the colonization status will be provided by improving specificity for the diagnosis of $\mathrm{PcP}$ vs. colonization in lower respiratory tract samples like bronchoalveolar lavage (BAL), endotracheal aspirate (ETA), and in less invasive samples like sputum or nasopharyngeal aspirates (NA) and oral lavage. Such improvements will enable the identification of pathogenic effects and diagnosis of emerging Pneumocystis pneumonia in the non-HIV-related population (Robert-Gangneux et al., 2014; Fauchier et al., 2016; Sarasombath et al., 2021) and the non-invasive diagnosis of Pneumocystis in newborns and young infants.

Recently, the complete genome assemblies for Pneumocystis species infecting humans ( $P$. jirovecii), rats ( $P$. carinii), and mice
(P. murina) have been reported and are available from the NCBI Umbrella project PRJNA223519 (Ma et al., 2016). The most significantly enriched domains in $P$. jirovecii and $P$. carinii are the multicopy gene families encoding the major surface glycoproteins (Msg), which are absent in another fungal species analyzed by Ma et al. (2016). In the aforementioned study based on sequence similarity, Msg proteins were classified into five families: Msg-A, -B, -C, -D, and -E (Ma et al., 2016), where Msg-A proved to be by far the largest family. Our study aims to develop and validate a more sensitive new real-time single-round PCR method based on simultaneously amplifying two regions, the mtLSUrRNA and the Msg-A multicopy gene family, a nested-PCR alternative for low levels of Pneumocystis diagnosis. We aim to increase specificity by enhancing the recognition of $P$. jirovecii-specific targets and reducing false-positive results caused by contamination or crossreactions.

\section{MATERIALS AND METHODS}

\section{Clinical Samples and DNA Extraction Protocol}

This study considered 73 biological samples, including 60 lung autopsies of healthy infants who died due to sudden death (LUNG 1-54, LUNG 1650, 1783, 895, 2604, 73B, and 65B), seven nasal aspirates (NA 102, 113, 119, 143, 150, 501, 502), three BAL (BAL FQ773, 953 and C965) and three gargles (G 05v4, $04 \mathrm{v} 5$, and $02 \mathrm{v} 5$ ). Diagnosis of autopsy samples from infants who suffered sudden unexpected death was established based on clinical history, macroscopic examination and dissection with histological sampling of major organs and laboratory tests including toxicology determinations. Postmortem bacterial cultures were not considered and detection of positive cases of respiratory viruses were excluded. Samples were categorized as $P$. jirovecii-positive based on the presence of $P$. jirovecii specific DNA by nested-PCR and, in the case of lung samples, also by the immunofluorescence-based detection of ascus forms with MERIFLUOR Pneumocystis (Meridian Bioscience).

The Ethics Commission for Studies in Human Subjects of the University of Chile School of Medicine approved this study under protocol CEISH \#092-2013. All methods were performed following the principles of the Declaration of Helsinki for medical research involving human subjects and following the relevant local guidelines and regulations.

Total DNA was extracted and purified following method B described by Ruiz-Ruiz et al. (2019). Said method consisted of DNA extraction with the QIAamp DNA Mini kit (Qiagen) and previous additional steps, including pre-treatment (samples were homogenized by magnetic stirrer agitation in sterile PBS), bead-beating and Phenol: Chloroform: Isoamyl alcohol steps. All manipulations were made inside a biosafety cabinet using new sterile equipment. DNA extraction was carried out in groups of five samples and each group with its respective extraction controls. In the case of lung tissue samples, it is well-known that Pneumocystis distribution in the competent host lung is focal in lung samples. However, previous studies showed that sampling $3 \%$ of the weight of the right upper lobe is enough to obtain 
excellent sensitivity in order to identify low levels of Pneumocystis (Vargas et al., 2017). DNA concentrations of all samples were measured using the Qubit double-stranded DNA (dsDNA) BR assay kit (Invitrogen) on a Qubit 4.0 Fluorometer (Invitrogen).

\section{mtLSUrRNA Nested-PCR}

Pneumocystis DNA was detected first using the nested-PCR procedure, amplifying part of the gene coding for mitochondrial large subunit ribosomal RNA (mtLSUrRNA) (Wakefield et al., 1990). The first round of nested-PCR was performed using the oligonucleotide primers pAZ102-E and pAZ102-H, utilizing 5 $\mu \mathrm{L}$ of DNA preparation. Amplification was performed under the following conditions: $94^{\circ} \mathrm{C}$ for $5 \mathrm{~min}, 94^{\circ} \mathrm{C}$ for $1.0 \mathrm{~min}, 55^{\circ} \mathrm{C}$ for $1.0 \mathrm{~min}$, and $72^{\circ} \mathrm{C}$ for $2.0 \mathrm{~min}$ for 40 cycles. The second round of nested-PCR was performed using pAZ102-X and pAZ102-Y primers, which are internal to the first set of primers and specific for $P$. jirovecii taking $1 \mu \mathrm{L}$ from the first-round PCR product under the following PCR conditions: $94^{\circ} \mathrm{C}$ for $5 \mathrm{~min}, 94^{\circ} \mathrm{C}$ for $1.0 \mathrm{~min}, 56^{\circ} \mathrm{C}$ for $1.0 \mathrm{~min}$, and $72^{\circ} \mathrm{C}$ for $2.0 \mathrm{~min}$ for 10 cycles and $94^{\circ} \mathrm{C}$ for $1.0 \mathrm{~min}, 64^{\circ} \mathrm{C}$ for $1.0 \mathrm{~min}$ and $72^{\circ} \mathrm{C}$ for $2.0 \mathrm{~min}$ for 30 cycles. Samples were categorized as follows: (a) Pneumocystispositive, when the $P$. jirovecii DNA specific 267 bp DNA band was obtained by nested-PCR in the sample, or one or more analyzed lobes in the case of lung samples, and (b) Pneumocystisnegative if no P. jirovecii DNA was documented. Pneumocystisnegative samples were analyzed twice, starting from the tissue or fluid. Controls were run simultaneously with samples. Positive controls were lung tissue DNA from patients with PCP. Negative controls such as water and DNA from Pneumocystis-negative lung tissue were used as templates in the first round PCR to control contamination, which was used de novo as a template for the second round PCR (negative controls). DNA amplification was performed twice for each sample. The PCR product was analyzed by electrophoresis in a $2 \%$ agarose gel and visualized with GelRed ${ }^{\circledR}$ (Biotium).

\section{Primer Design for Real-Time Msg-A/mtLSUrRNA PCR}

The two pairs of primers (Msg-AF-Msg-AR and MlsF-MlsR) were designed based on the conserved sequences in $P$. jirovecii Msg-A and mtLSUrRNA genes using the Primer Express software (Applied Biosystems) and a multiple alignment of all MsgA DNA sequences obtained from the original publication by Ma et al. (2016) (accession code in the NCBI BioProject database PRJNA223510) or a representative number of all the complete sequences of the $P$. jirovecii mtLSUrRNA gene deposited in the NCBI and GenBank databases, respectively. These multiple alignments were obtained with the ClustalW program (Thompson et al., 2002). For the Msg alignment, the primer set contained 179 genes that were annotated in the $P$. jirovecii genome and represented all the existing variation of paralogs in a genome. The nucleotide sequence of the primer set Msg-AF and Msg-AR contained three degenerate positions each (Supplementary Figure 1). The target specificity of the new pairs of primers was evaluated with total DNA from culture of other lung pathogens such as Aspergillus fumigatus, Candida albicans, or Cryptococcus neoformans, which are involved in other lung infections.

\section{Real-Time Msg-A/mtLSUrRNA PCR Conditions}

Real-time PCR was performed in a LightCycler ${ }^{\circledR} 480$ System (Roche Molecular Diagnostics) using $10 \mu \mathrm{L}$ of a reaction mix that contained $5 \mu \mathrm{L}$ of LightCycler ${ }^{\circledR}$ FastStart DNA Master Plus SYBR Green I (Roche Diagnostics), $1.8 \mu \mathrm{L}$ of DNase-free water, $0.3 \mu \mathrm{M}$ of each primer and $2 \mu \mathrm{L}$ of total DNA extraction (10 ng DNA/ $\mu \mathrm{L})$. Control samples in each run included total DNA from lung tissue Pneumocystis-negative, water instead of total DNA extract. Each sample was analyzed in duplicate in each independent real-time PCR assay. All the DNA extractions were adjusted to approximately $10 \mathrm{ng} / \mu \mathrm{L}$ and then measured with the Qubit 4 Fluorometer (Invitrogen) to allow uniformity in sample collection. Cycling conditions included incubation at $95^{\circ} \mathrm{C}$ for $10 \mathrm{~min}$ and 45 cycles of $95^{\circ} \mathrm{C}$ for $10 \mathrm{~s}, 58^{\circ} \mathrm{C}$ for $10 \mathrm{~s}$, and $72^{\circ} \mathrm{C}$ for $15 \mathrm{~s}$ (when we took fluorescence measurements). The melting curves analysis was performed with the LightCycler platform software, displaying the first derivative of the fluorescence intensity vs. the temperature. Synthesis of DNA products of the expected size was confirmed by melting curve analysis and electrophoretic separation in a 2\% agarose gel and staining with GelRed ${ }^{\circledR}$ (Biotium). The LightCycler software plotted the fluorescence intensity against the number of cycles and provided the threshold cycle $(C t)$ value using the automatic method for each run. Sensitivity, specificity, positive predictive value (PPV), and negative predictive value (NPV) were reported only for the 60 lung autopsies of healthy infants who died due to sudden death (LUNG 1-54, LUNG 1650, 1783, 895, 2604, 73B and 65B) considering the nested-PCR as the reference method.

\section{Real-Time PCR Protocol From Rudramurthy's Study}

We compared the new real-time PCR protocol with the protocol developed by Rudramurthy et al. (2018), comprising a realtime quantitative PCR using Msg genes [primer pairs described by Linssen et al. (2006), F 5'-CAA AAA TAA CAY TSA CAT CAA CRA GG-3' and R 5'-AAA TCA TGA ACG AAA TAA CCATTG C-3']. For this comparative study, all Pneumocystispositive samples were included. Because our new protocol simultaneously amplifies two regions, in addition to comparing Rudramurthy's protocol with our Msg-A/mtLSUrRNA real-time PCR, we have also compared them with the primer set Msg-A in only one group of samples (LUNG 19-54). The real-time PCRs were performed in a LightCycler ${ }^{\circledR} 480$ System (Roche Molecular Diagnostics) using $10 \mu \mathrm{L}$ of a reaction mix that contained 5 $\mu \mathrm{L}$ of LightCycler ${ }^{\circledR}$ FastStart DNA Master Plus SYBR Green I (Roche Diagnostics), $1.8 \mu \mathrm{L}$ of DNase-free water (in the case of using both pairs of primers) or $2.4 \mu \mathrm{L}$ of DNase-free water (using only the primers that amplify Msg-A), $0.3 \mu \mathrm{M}$ of each primer and $2 \mu \mathrm{L}$ of total DNA extraction (10 ng DNA/ $\mu \mathrm{L}$ ). Cycling conditions were performed according to Rudramurthy et al. (2018). Control samples in each run included total DNA from lung tissue Pneumocystis-negative and water instead of total 
DNA extract. Each sample was analyzed in duplicate. Statistical comparisons between the two real-time PCR methods were made using paired $t$-tests.

\section{RESULTS}

\section{Real-Time Msg-A/mtLSUrRNA PCR Optimization}

The primer sets were designed based on multiple alignments. The nucleotide sequence of the primer set Msg-AF- Msg-AR contained three degenerate positions each to amplify many related genes of the Msg-A family (Supplementary Figure 1).

For the PCR cycling conditions, we selected the program providing the most sensitive detection. Although low DNA concentration was observed in some samples, we noted that better results were obtained using diluted DNA extractions adjusted to $10 \mathrm{ng} / \mu \mathrm{L}$ than non-diluted DNA extraction ranging from 2118 to $41.3 \mathrm{ng} / \mu \mathrm{L}$. In fact, for some samples (LUNG 19, LUNG 21, LUNG 26, and LUNG 46), no exponential amplification was observed with the undiluted DNA extraction (Supplementary Figure 2). Generally, for the LightCycler ${ }^{\circledR} 480$ System, Roche recommends the use of DNA concentrations ranging from 5 to $30 \mathrm{ng}$ for real-time PCR.

The specificity of Msg-A/mtLSUrRNA PCR amplification was confirmed by melting curves and amplified products in all $P$. jirovecii-positive samples. It is worth mentioning that each pair of primers amplified a region of similar size and had similar annealing temperatures. The sufficiently different percentage of GC nucleotides (51\% GC for the Msg-A region and 36\% for the mtLSUrRNA region) was also important in selecting the region to design the primers, allowing the visualization of two separate melting curves according to their different $\mathrm{Tm}$ values. The results indicated whether one or two regions were amplified. The first derivative of the melting curve in the new MsgA/mtLSUrRN real-time PCR showed two peaks with melting temperatures ranging from 81.00 to $83.00^{\circ} \mathrm{C}(\mathrm{Msg}-\mathrm{A})$ and from 74.00 to $75.00^{\circ} \mathrm{C}$ (mtLSUrRNA), with the exception of all three nasal aspirate samples (NA 501, 502, and 113), in which only the melting curve of the Msg-A gene family was observed. Meanwhile, a single band with the expected size (around $117 \mathrm{bp}$ ) was observed for all the Pneumocystis-positive samples.

We did not observe any amplification product or melting curve profile when the primers were evaluated using other pathogens such as Aspergillus fumigatus, Candida albicans, and Cryptococcus neoformans, occasionally encountered in the lung.

\section{Comparison of the Nested-PCR and the Msg-A/mtLSUrRNA PCR}

To validate our new real-time Msg-A/mtLSUrRNA PCR protocol, we compared it with the results obtained by the nestedPCR (mtLSUrRNA). Lung samples from LUNG 19 to 54, LUNG $1650,1783,895,2604,73 \mathrm{~B}$ and 65B, nasal aspirates NA 119, 143 and 150, BAL FQ773, 953 and C965 and gargles G 05v4, 04v5 and 02v5 were categorized as $P$. jirovecii-positive given the presence of $P$. jirovecii specific DNA by nested-PCR (54/73 samples) and ascus forms by immunofluorescence (only in lung samples) (Table 1). All samples identified as $P$. jirovecii-positive using the nested-PCR method were also classified as Pneumocystispositive using the real-time Msg-A/mtLSUrRNA PCR protocol with $C t$ values ranging from 25.30 to 40.00 (Table 1). However, some positive samples presented low $P$. jirovecii loads as no PCR amplification or weak bands were observed (samples LUNG 20, 22, 24, 30, 35, 39, 40, 45, 48, 52, and 53) in the first round of nested-PCR (mtLSUrRNA) (Supplementary Figure 3). For the real-time Msg-A/mtLSUrRNA PCR, these samples presented amplification curves with $C t$ values ranging from 35.40 to 40.00 (Table 1) and two peaks of melting curves for the two amplified genes. In addition, three samples of nasal aspirates (NA 501, 502, and 113) that were negative with the nested-PCR were positive with Msg-A/mtLSUrRNA PCR protocol observing only the melting curve of the Msg-A gene family (Supplementary Figure 4). The remaining Pneumocystisnegative samples were also confirmed negative by the MsgA/mtLSUrRNA PCR. Considering the mtLSUrRNA nested-PCR as the reference method, the sensitivity, specificity, PPV and NPV values of the new real-time Msg-A/mtLSUrRNA PCR protocol were $100,100,100$, and $100 \%$, respectively, for the lung tissue samples group.

\section{Comparison of Msg-A/mtLSUrRNA PCR and Rudramurthy's PCR}

To evaluate the efficiency of our Msg-A/mtLSUrRNA PCR protocol, we analyzed all Pneumocystis-positive samples in this study using the PCR protocol published by Rudramurthy et al. (2018). As shown in Table 1, the results differ between the two PCR protocols. Our PCR protocol presents higher sensitivity than the protocol reported by Rudramurthy et al. (2018). For $P$. jirovecii-positive samples, the $C t$ values obtained with our PCR protocol were significantly lower than those obtained by Rudramurthy's protocol (33.75 \pm 4.0 vs. $39.18 \pm 1.7$, $p$-value < 0.05) (Table 1). Ten Pneumocystis-positive samples identified with our Msg-A/mtLSUrRNA PCR were classified negative with the PCR protocol published by Rudramurthy et al. (2018) (Supplementary Figure 5). Furthermore, because our new Msg-A/mtLSUrRNA real-time PCR protocol simultaneously amplifies two regions while the Rudramurthy et al. (2018) protocol amplifies only one, we have also compared them by amplifying the Msg region alone with our primer set Msg-A in a group of samples (LUNG 19-54). The comparison of our Msg-A primer pairs separately also obtained lower $C t$ values than those obtained with Rudramurthy's (Msg) protocol (30.1 \pm 2.36 vs. $38.01 \pm 2.22$, respectively, $p$-value $<0.05)$, thus demonstrating better optimization in the design of these new primers (Table 1).

\section{DISCUSSION}

In this work, we validated a laboratory-developed real-time assay for reliable detection of $P$. jirovecii. Our results showed that our Msg-A/mtLSUrRNA PCR provides sensitive data in samples containing low $P$. jirovecii loads and presents both high sensitivity and specificity. This method has several advantages: firstly, both 
TABLE 1 | Detection of $P$. jirovecii in samples by real-time Msg-A/mtLSUrRNA PCR compared to those amplified with the protocol developed by Rudramurthy et al. (2018) or by nested-PCR.

\begin{tabular}{|c|c|c|c|c|c|}
\hline \multirow[b]{2}{*}{ Sample } & \multicolumn{2}{|c|}{ Average $C t^{a} \pm S D^{b}$} & \multicolumn{2}{|c|}{ Nested-PCR } & \multirow[t]{2}{*}{ IF } \\
\hline & $\begin{array}{l}\text { Real-time } \\
\text { PCR } \\
\text { Rudramurthy } \\
\text { et al. (2018) }\end{array}$ & $\begin{array}{c}\text { Real-time } \\
\text { Msg- } \\
\text { A/mtLSUrRNA } \\
\text { PCR }\end{array}$ & 1st Round & 2nd Round & \\
\hline LUNG 19 & $36.7 \pm 0.1$ & $26.3 \pm 0.2$ & + & + & + \\
\hline LUNG 20 & $>40.0 \pm 0$ & $36.4 \pm 0.1$ & + & + & + \\
\hline LUNG 21 & $33.8 \pm 0.3$ & $25.3 \pm 0.1$ & + & + & + \\
\hline LUNG 22 & NEG & $35.8 \pm 0.3$ & - & + & - \\
\hline LUNG 23 & $>40.0 \pm 0$ & $33.7 \pm 0.3$ & + & + & + \\
\hline LUNG 24 & NEG & $35.4 \pm 0.3$ & + & + & + \\
\hline LUNG 25 & $38.2 \pm 0.1$ & $28.9 \pm 0.2$ & + & + & + \\
\hline LUNG 26 & $35.6 \pm 0.2$ & $28.7 \pm 0.1$ & + & + & + \\
\hline LUNG 27 & $>40.0 \pm 0$ & $30.3 \pm 0.1$ & + & + & + \\
\hline LUNG 28 & $>40.0 \pm 0$ & $34.3 \pm 0.2$ & + & + & + \\
\hline LUNG 29 & $>40.0 \pm 0$ & $35.8 \pm 0.3$ & + & + & + \\
\hline LUNG 30 & NEG & $37.2 \pm 0.1$ & - & + & - \\
\hline LUNG 31 & $>40.0 \pm 0$ & $32.1 \pm 0.2$ & + & + & + \\
\hline LUNG 32 & $38.2 \pm 0.3$ & $29.5 \pm 0.3$ & + & + & + \\
\hline LUNG 33 & $>40.0 \pm 0$ & $32.6 \pm 0.2$ & + & + & + \\
\hline LUNG 34 & $36.6 \pm 0.3$ & $28.4 \pm 0.3$ & + & + & + \\
\hline LUNG 35 & NEG & $37.9 \pm 0.4$ & - & + & - \\
\hline LUNG 36 & $>40.0 \pm 0$ & $32.6 \pm 0.4$ & + & + & + \\
\hline LUNG 37 & $>40.0 \pm 0$ & $33.3 \pm 0.3$ & + & + & + \\
\hline LUNG 38 & $>40.0 \pm 0$ & $30.5 \pm 0.2$ & + & + & + \\
\hline LUNG 39 & $>40.0 \pm 0$ & $37.6 \pm 0.5$ & + & + & - \\
\hline LUNG 40 & $>40.0 \pm 0$ & $35.4 \pm 0.3$ & + & + & + \\
\hline LUNG 41 & $>40.0 \pm 0$ & $30.9 \pm 0.2$ & + & + & + \\
\hline LUNG 42 & $>40.0 \pm 0$ & $32.7 \pm 0.3$ & + & + & + \\
\hline LUNG 43 & $35.6 \pm 0$ & $26.8 \pm 0.2$ & + & + & + \\
\hline LUNG 44 & $>40.0 \pm 0$ & $35.1 \pm 0.3$ & + & + & + \\
\hline LUNG 45 & $>40.0 \pm 0$ & $36.8 \pm 0.4$ & + & + & + \\
\hline LUNG 46 & $37.0 \pm 0.1$ & $30.2 \pm 0.3$ & + & + & + \\
\hline LUNG 47 & $>40.0 \pm 0$ & $34.3 \pm 0.3$ & + & + & + \\
\hline LUNG 48 & NEG & $38.3 \pm 0.5$ & + & + & + \\
\hline LUNG 49 & $>40.0 \pm 0$ & $34.8 \pm 0.4$ & + & + & + \\
\hline LUNG 50 & $39.2 \pm 0.2$ & $30.0 \pm 0.3$ & + & + & + \\
\hline LUNG 51 & $>40.0 \pm 0$ & $33.2 \pm 0.2$ & + & + & + \\
\hline LUNG 52 & $>40.0 \pm 0$ & $>40.0 \pm 0$ & + & + & - \\
\hline LUNG 53 & $>40.0 \pm 0$ & $35.8 \pm 0.4$ & + & + & + \\
\hline LUNG 54 & $>40.0 \pm 0$ & $33.4 \pm 0.3$ & + & + & + \\
\hline LUNG 1650 & NEG & $>40.0 \pm 0$ & + & + & + \\
\hline LUNG 1783 & $>40.0 \pm 0$ & $38.4 \pm 0.5$ & + & + & - \\
\hline LUNG 895 & $>40.0 \pm 0$ & $38.8 \pm 0.6$ & + & + & + \\
\hline LUNG 2604 & $>40.0 \pm 0$ & $31.4 \pm 0.2$ & + & + & + \\
\hline LUNG 73B & $>40.0 \pm 0$ & $32.3 \pm 0.2$ & + & + & + \\
\hline LUNG 65B & $33.2 \pm 0.7$ & $25.7 \pm 0.4$ & + & + & + \\
\hline G 05v4 & NEG & $39.56 \pm 0.3$ & + & + & NA \\
\hline G 04v5 & $>40.0 \pm 0$ & $34.79 \pm 0.5$ & + & + & NA \\
\hline G 02v5 & NEG & $>40.0 \pm 0$ & + & + & NA \\
\hline NA 113 & $>40.0 \pm 0$ & $35.9 \pm 0.3$ & - & - & NA \\
\hline NA 119 & $>40.0 \pm 0$ & $29.7 \pm 0.1$ & + & + & NA \\
\hline
\end{tabular}

(Continued)
TABLE 1 | (Continued)

\begin{tabular}{|c|c|c|c|c|c|}
\hline \multirow[b]{2}{*}{ Sample } & \multicolumn{2}{|c|}{ Average $C t^{a} \pm S D^{b}$} & \multicolumn{2}{|c|}{ Nested-PCR } & \multirow[t]{2}{*}{ IF } \\
\hline & $\begin{array}{l}\text { Real-time } \\
\text { PCR } \\
\text { Rudramurthy } \\
\text { et al. (2018) }\end{array}$ & $\begin{array}{c}\text { Real-time } \\
\text { Msg- } \\
\text { A/mtLSUrRNA } \\
\text { PCR }\end{array}$ & 1st Round & 2nd Round & \\
\hline NA 143 & $>40.0 \pm 0$ & $34.9 \pm 0.5$ & + & + & NA \\
\hline NA 501 & NEG & $>40.0 \pm 0$ & - & - & NA \\
\hline NA 502 & NEG & $>40.0 \pm 0$ & - & - & NA \\
\hline NA 150 & $>40.0 \pm 0$ & $27.8 \pm 0.2$ & + & + & NA \\
\hline BAL FQ773 & $>40.0 \pm 0$ & $34.3 \pm 0.5$ & + & + & NA \\
\hline BAL 953 & $>40.0 \pm 0$ & $35.9 \pm 0.4$ & + & + & NA \\
\hline BAL C965 & $>40.0 \pm 0$ & $33.1 \pm 0.3$ & + & + & NA \\
\hline
\end{tabular}

amplification and detection steps are performed in a closed system, thus reducing the potential for contamination; secondly, it is rapid, requiring less hands-on time and allowing sameday sample analysis, with detection performed in just 3-4 h, including DNA extraction; thirdly, it is as sensitive as nestedPCR, which is considered one of the main protocols used for $P$. jirovecii detection.

Nested-PCR is based on amplifying the gene coding for Pneumocystis mitochondrial large-subunit ( $\mathrm{mt} \mathrm{LSU}$ ) rRNA gene, which is present in multicopy and whose number is augmented by the number of mitochondria in fungi (Valero et al., 2016). Using multicopy genes as a target for PCR enhances its sensitivity. Robberts et al. (2007) showed that nested-PCR had the highest sensitivity to detect $P$. jirovecii in clinical samples from South African patients compared to nine other molecular assays. Although the method developed by Wakefield et al. (1990) and Wakefield (1996) became the benchmark and was taken on board for the epidemiologic investigation of Pneumocystis data generated by nested-PCR, it requires multiple controls and extreme care to avoid contamination. In effect, scrupulous laboratory procedures must be implemented to prevent sample contamination and ensure the absence of PCR inhibitors by applying appropriate controls.

Besides the potential problems of laboratory contamination, nested-PCR is also complicated, labor-intensive, and timeconsuming (requiring 2 days). In this light, the real-time PCR method seems to be a more advantageous technique, as it is both rapid and sensitive. For the real-time Msg-A/mtLSUrRNA PCR protocol developed here, the specificity of the primers was further supported by the presence of two single fluorescence peaks in the melting curves. The slight variation recorded in the Tm values of each melting curve can be explained by the GC content variability within the amplified sequences in the samples (Ririe et al., 1997). Moreover, primer specificity was further corroborated by their failure to amplify DNA extracted from other pathogens typically isolated in the lung. Generally, probe-based PCR is usually used for clinical diagnosis since it is 
considered more specific than SYBR Green PCR. However, the use of SYBR Green is less expensive than probe synthesis, and the lower specificity of the SYBR Green affords an advantage when amplifying a heterogeneous region (Papin et al., 2004). A few mismatches within the region targeted by the probes can miss the hybridization of some sequence variants, leading to false negatives (Read et al., 2001; Varga and James, 2005). This point is of primary importance as the amplified regions used for $P$. jirovecii detection, such as the mtLSU rRNA gene or the Msg-A gene, are present in multicopy and could evolve independently. Extensive variations were observed in the Msg-A repertoire among $P$. jirovecii isolates partially generated by recombination (Kutty et al., 2008), which provide a large potential for antigenic variation and presumably facilitate evasion of immune responses in hosts (Garbe and Stringer, 1994; Wright et al., 1995). In this context, the design of primers for specific amplification of Msg-A also presents a challenge. For this reason, both Msg-A primers designed in this study contain three degenerate positions to improve the match and to amplify the greatest number of Msg-A genes showing strong specificity and amplification efficiency. On the other hand, the selection of the region for which the primer pairs were designed contained a sufficiently different percentage of GC nucleotides enabling two separate melting curves to be visualized due to their different Tm values or just one of them in the even that only one of the two regions was amplified. The comparison of the nested-PCR and the Msg-A/mtLSUrRNA PCR showed that three samples of nasal aspirates (NA 501, 502, and 113) were negative with the nested-PCR positive with MsgA/mtLSUrRNA PCR protocol observing only the melting curve of the Msg-A gene family. This may be due to very low titers of $P$. jirovecii, with the detection of only the most abundant target in this sample or the existence of variability in the region of that gene where the annealing of primers takes place. Multiplex PCR reduces the number of reactions needed to test a sample for different targets and helps save time and money (Sint et al., 2012).

The comparison of Msg-A/mtLSUrRNA PCR and Rudramurthy's PCR showed that our PCR protocol presents higher sensitivity at lower $C t$ values. In HIV patients, Rudramurthy et al. (2018) estimated that PCR amplification at a $C t$ value less than, or equal to, 25 were positive for PcP, and those that gave $C t$ values greater or equal than 26 were considered as colonized. In our study, the $C t$ values were greater than or equal to 26, which could be correlated with the Pneumocystis colonized status of most of our samples.

The proposed real-time Msg-A/mtLSUrRNA PCR is of value due to its increased sensitivity and specificity by improved recognition of two $P$. jirovecii-specific targets simultaneously, namely the mtLSUrRNA and the Msg-A multicopy gene family

\section{REFERENCES}

Alanio, A., Desoubeaux, G., Sarfati, C., Hamane, S., Bergeron, A., Azoulay, E., et al. (2011). Real-time PCR assay-based strategy for differentiation between active Pneumocystis jirovecii pneumonia and colonization in immunocompromised patients. Clin. Microbiol. Infect. 17, 1531-1537.

Alanio, A., Gits-Muselli, M., Mercier-Delarue, S., Dromer, F., and Bretagne,

S. (2016). Diversity of Pneumocystis jirovecii during infection revealed by and its reduction of false positive results caused by contamination or cross-reactions. Furthermore, it provides an alternative to nested-PCR for low levels of Pneumocystis diagnosis, performing detection in just 3-4 h, including DNA extraction.

\section{DATA AVAILABILITY STATEMENT}

The original contributions presented in the study are included in the article/Supplementary Material, further inquiries can be directed to the corresponding author/s.

\section{ETHICS STATEMENT}

The studies involving human participants were reviewed and approved by the Ethics Commission for Studies in Human Subjects of the University of Chile School of Medicine approved this study under protocol CEISH \#092-2013. Written informed consent to participate in this study was provided by the participants' legal guardian/next of kin.

\section{AUTHOR CONTRIBUTIONS}

SR-R, CP, and AM: conceptualization and writing. SR-R, CP, NP, RB, GG, VS, MG, and PB: methodology. SR-R and CP: validation and investigation and data curation. SR-R, CP, and NP: formal analysis. SR-R, CP, FM, SV, VP-B, and AM: writing-review and editing. AM: supervision. SV and AM: funding acquisition. All authors have read and agreed to the published version of the manuscript.

\section{FUNDING}

This research was funded by the ERANet LAC (ELAC2014/HID0254), the National Fund for Science and Technology (Fondecyt, Chile) (1140412), the Spanish Ministry of Science and Innovation (PID2019-105969GB-I00), Generalitat Valenciana (Spain) (Prometeo/2018/A/133), and co-financed by the European Regional Development Fund (ERDF, EU).

\section{SUPPLEMENTARY MATERIAL}

The Supplementary Material for this article can be found online at: https://www.frontiersin.org/articles/10.3389/fmicb. 2021.787554/full\#supplementary-material

ultra-deep pyrosequencing. Front. Microbiol. 7:733. doi: 10.3389/fmicb.2016. 00733

Bossart, S., Mühlethaler, K., Garzoni, C., and Furrer, H. (2020). Is real time PCR preferable to the direct immunofluorescence in the diagnosis of Pneumocystis jirovecii pneumonia in HIV-infected patients? BMC Res. Notes 13:235.

Chabé, M., Khalife, S., Gantois, N., Even, G., and Audebert, C. (2014). An improved single-round PCR leads to rapid and highly sensitive detection of Pneumocystis spp. Med. Mycol. 52, 841-846. doi: 10.1093/mmy/myu032 
Chotiprasitsakul, D., Pewloungsawat, P., Setthaudom, C., Santanirand, P., and Pornsuriyasak, P. (2020). Performance of real-time PCR and immunofluorescence assay for diagnosis of Pneumocystis pneumonia in real-world clinical practice. PLoS One 15:e0244023. doi: 10.1371/journal.pone. 0244023

Dellière, S., Gits-Muselli, M., White, P. L., Mengoli, C., Bretagne, S., and Alanio, A. (2019). Quantification of Pneumocystis jirovecii: cross-platform comparison of one qPCR assay with leading platforms and six master mixes. J. Fungi 6:9.

Dunaiski, C. M., Janssen, L., Erzinger, H., Pieper, M., Damaschek, S., and Schildgen, O. (2018). Inter-specimen imbalance of mitochondrial gene copy numbers predicts clustering of Pneumocystis jirovecii isolates in distinct subgroups. J. Fungi 4:84. doi: 10.3390/jof 4030084

Esteves, F., Gaspar, J., De Sousa, B., Antunes, F., Mansinho, K., and Matos, O. (2011). Clinical relevance of multiple single-nucleotide polymorphisms in Pneumocystis jirovecii Pneumonia: development of a multiplex PCR-singlebase-extension methodology. J. Clin. microbiol. 49, 1810-1815.

Fauchier, T., Hasseine, L., Gari-Toussaint, M., Casanova, V., Marty, P. M., and Pomares, C. (2016). Detection of Pneumocystis jirovecii by quantitative PCR to differentiate colonization and pneumonia in immunocompromised HIVpositive and HIV-negative patients. J. Clin. Microbiol. 54, 1487-1495. doi: 10. 1128/JCM.03174-15

Fraczek, M. G., Ahmad, S., Richardson, M., Kirwan, M., Bowyer, P., Denning, D. W., et al. (2019). Detection of Pneumocystis jirovecii by quantitative realtime PCR in oral rinses from Pneumocystis pneumonia asymptomatic human immunodeficiency virus patients. J. Med. Mycol. 29, 107-111. doi: 10.1016/j. mycmed.2019.04.001

Garbe, T. R., and Stringer, J. R. (1994). Molecular characterization of clustered variants of genes encoding major surface antigens of human Pneumocystis carinii. Infect Immun. 62, 3092-3101. doi: 10.1128/iai.62.8.3092-3101

Gits-Muselli, M., White, P. L., Mengoli, C., Chen, S., Crowley, B., Dingemans, G., et al. (2020). The Fungal PCR Initiative's evaluation of in-house and commercial Pneumocystis jirovecii qPCR assays: toward a standard for a diagnostics assay. Med. Mycol. 58, 779-788. doi: 10.1093/mmy/myz115

Kutty, G., Maldarelli, F., Achaz, G., and Kovacs, J. A. (2008). Variation in the major surface glycoprotein genes in Pneumocystis jirovecii. J. Infect. Dis. 198, 741-749.

Linssen, C. F. M., Jacobs, J. A., Beckers, P., Templeton, K. E., Bakkers, J., Kuijper, E. J., et al. (2006). Inter-laboratory comparison of three different real-time PCR assays for the detection of Pneumocystis jirovecii in bronchoalveolar lavage fluid samples. J. Med. Microbiol. 55, 1229-1235. doi: 10.1099/jmm.0.46552-0

Ma, L., Chen, Z., Huang da, W., Kutty, G., Ishihara, M., Wang, H., et al. (2016). Genome analysis of three Pneumocystis species reveals adaptation mechanisms to life exclusively in mammalian hosts. Nat. Commun. 7:10740.

Montesinos, I., Delforge, M. L., Ajjaham, F., Brancart, F., Hites, M., Jacobs, F., et al. (2017). Evaluation of a new commercial real-time PCR assay for diagnosis of Pneumocystis jirovecii pneumonia and identification of dihydropteroate synthase (DHPS) mutations. Diagn. Microbiol. Infect. Dis. 87, 32-36. doi: 10. 1016/j.diagmicrobio.2016.10.005

Morilla, R., González-Magaña, A., Friaza, V., de Armas, Y., Medrano, F. J., Calderón, E. J., et al. (2019). Genetic polymorphisms of superoxide dismutase locus of Pneumocystis jirovecii in Spanish population. Front. Public Health 7:292. doi: 10.3389/fpubh.2019.00292

Morris, A., Wei, K., Afshar, K., and Huang, L. (2008). Epidemiology and clinical significance of Pneumocystis colonization. J. Infect. Dis. 97, 10-17.

Özkoç, S., Köker, M., Önder, M., and Delibaş, S. B. (2016). Prevalence of Pneumocystis jirovecii colonization in autopsy cases in Turkey. J. Med. Microbiol. 65, 1152-1157. doi: 10.1099/jmm.0.000337

Papin, J. F., Vahrson, W., and Dittmer, D. P. (2004). SYBR green-based real-time quantitative PCR assay for detection of West Nile Virus circumvents falsenegative results due to strain variability. J. Clin. Microbiol. 42, 1511-1518. doi: 10.1128/JCM.42.4.1511-1518.2004

Ponce, C. A., Gallo, M., Bustamante, R., and Vargas, S. L. (2010). Pneumocystis colonization is highly prevalent in the autopsied lungs of the general population. Clin. Infect. Dis. 50, 347-353.

Read, S. J., Mitchell, J. J., and Fink, C. G. (2001). LightCycler multiplex PCR for the laboratory diagnosis of common viral infections of the central nervous system. J. Clin. Microbiol. 39, 3056-3059. doi: 10.1128/JCM.39.9.3056-3059.2001

Ririe, K., Rasmussen, R. P., and Wittwer, C. T. (1997). Product differentiation by analysis of DNA melting curves during the polymerase chain reaction. Anal. Biochem. 245, 154-160.
Robberts, F. J., Liebowitz, L. D., and Chalkley, L. J. (2007). Polymerase chain reaction detection of Pneumocystis jirovecii: evaluation of 9 assays. Diagn. Microbiol. Infect. Dis. 58, 385-392. doi: 10.1016/j.diagmicrobio.2007.02.014

Robert-Gangneux, F., Belaz, S., Revest, M., Tattevin, P., Jouneau, S., Decaux, O., et al. (2014). Diagnosis of Pneumocystis jirovecii pneumonia in immunocompromised patients by real-time PCR: a 4-year prospective study. J. Clin. Microbiol. 52, 3370-3376. doi: 10.1128/JCM.014 80-14

Rudramurthy, S. M., Sharma, M., Sharma, M., Rawat, P., Ghosh, A., Venkatesan, L., et al. (2018). Reliable differentiation of Pneumocystis pneumonia from Pneumocystis colonisation by quantification of major surface glycoprotein gene using real-time polymerase chain reaction. Mycoses 61, 96-103.

Ruiz-Ruiz, S., Ponce, C. A., Pesantes, N., Bustamante, R., Gatti, G., San Martin, V., et al. (2019). New DNA extraction method for the detection of Pneumocystis in lung tissue samples of colonized individuals. OBM Genet. 3:11. doi: 10.21926/ obm.genet. 1901066

Sarasombath, P. T., Thongpiya, J., Chulanetra, M., Wijit, S., Chinabut, P., Ongrotchanakun, J., et al. (2021). Quantitative PCR to discriminate between pneumocystis pneumonia and colonization in HIV and Non-HIV immunocompromised patients. Front. Microbiol. 12:729193.

Sint, D., Raso, L., and Traugott, M. (2012). Advances in multiplex PCR: balancing primer efficiencies and improving detection success. Methods Ecol. Evol. 3, 898-905. doi: 10.1111/j.2041-210X.2012.00215.x

Thompson, J. D., Gibson, T. J., and Higgins, D. G. (2002). Multiple sequence alignment using ClustalW and ClustalX*. Curr. Protoc. Bioinformatics doi: 10. 1002/0471250953.bi0203s00

Valero, C., Buitrago, M. J., Gits-Muselli, M., Benazra, M., Sturny-Leclère, A., Hamane, S., et al. (2016). Copy number variation of mitochondrial DNA genes in Pneumocystis jirovecii according to the fungal load in BAL specimens. Front. Microbiol. 12:1413.

Varga, A., and James, D. (2005). Detection and differentiation of Plum pox virus using real-time multiplex PCR with SYBR Green and melting curve analysis: a rapid method for strain typing. J. Virol. Methods 123, 213-220.

Vargas, S. L., Ponce, C., Bustamante, R., Calderón, E., Nevez, G., De Armas, Y., et al. (2017). Importance of tissue sampling, laboratory methods, and patient characteristics for detection of Pneumocystis in autopsied lungs of non-immunosuppressed individuals. Eur. J. Clin. Microbiol. Infect. Dis. 36, 1711-1716. doi: 10.1007/s10096-017-3006-8

Wakefield, A. E. (1996). DNA sequences identical to Pneumocystis carinii f. sp. carinii and Pneumocystis carinii f. sp. hominis in samples of air spora. J. Clin. Microbiol. 34, 1754-1759. doi: 10.1128/JCM.34.7.1754-1759.1996

Wakefield, A. E., Pixley, F. J., Banerji, S., Sinclair, K., Miller, R. F., Moxon, E. R., et al. (1990). Detection of Pneumocystis carinii with DNA amplification. Lancet 336, 451-453. doi: 10.1016/0140-6736(90)92008-6

Wright, T. W., Bissoondial, T. Y., Haidaris, C. G., Gigliotti, F., and Haidaris, P. J. (1995). Isoform diversity and tandem duplication of the glycoprotein A gene in ferret Pneumocystis carinii. DNA Res. 2, 77-88. doi: 10.1093/dnares/2.2.77

Yang, S. L., Wen, Y. H., Wu, Y. S., Wang, M. C., Chang, P. Y., Yang, S., et al. (2020). Diagnosis of pneumocystis pneumonia by real-time PCR in patients with various underlying diseases. J. Microbiol. Immunol. Infect. 53, 785-790.

Conflict of Interest: The authors declare that the research was conducted in the absence of any commercial or financial relationships that could be construed as a potential conflict of interest.

Publisher's Note: All claims expressed in this article are solely those of the authors and do not necessarily represent those of their affiliated organizations, or those of the publisher, the editors and the reviewers. Any product that may be evaluated in this article, or claim that may be made by its manufacturer, is not guaranteed or endorsed by the publisher.

Copyright (C) 2022 Ruiz-Ruiz, Ponce, Pesantes, Bustamante, Gatti, San Martin, Gutierrez, Bórquez, Vargas, Magne, Calderón, Pérez-Brocal and Moya. This is an open-access article distributed under the terms of the Creative Commons Attribution License (CC BY). The use, distribution or reproduction in other forums is permitted, provided the original author(s) and the copyright owner(s) are credited and that the original publication in this journal is cited, in accordance with accepted academic practice. No use, distribution or reproduction is permitted which does not comply with these terms. 